\title{
El riesgo del parto vaginal en primigestas con episiotomía y sin episiotomía en el Hospital Nacional Juan José Fernández de El Salvador
}

\author{
Dr. Carlos Gamero \\ Jefe de Epidemiología del Hospital Nacional Zacamil del Ministerio de Salud Pública de El Salvador, \\ San Salvador
}

Recepcionado: 20 abril, 2013 / Aceptado: 14 de julio, 2013

\section{RESUMEN}

El presente estudio tiene como objetivo conocer la diferencia en el riesgo del parto vaginal en primigestas con episiotomía y sin episiotomía para los desgarros grado II, duración de la segunda fase del parto y del valor del APGAR del recién nacido al primer minuto, en los períodos 2003 al 2004 y del 2007 al 2008 en el Hospital Nacional Zacamil de El Salvador. Para ello, se realizó un estudio epidemiológico observacional, analítico de casos y controles de las primigestas que verificaron parto vaginal en los períodos del 2003 al 2004 que es el período en el cual no existían criterios para la realización de la episiotomía comparada con el período 2007 al 2008 en el cual ya se aplicaban criterios para la realización de la episiotomía. Se evaluaron los tres pilares fundamentales que tradicionalmente han justificado la realización de la episiotomía: disminución del riesgo de desgarros II o mayores, disminución de la duración del segundo período del trabajo del parto y el valor del APGAR al primer minuto. Entre los principales resultados, tenemos que la prevalencia de desgarros mayores de tipo II entre las pacientes primigestas con episiotomía y sin episiotomía no mostraron asociación significativa de factor de protección para el uso de episiotomía. De la misma manera no se demostró ninguna asociación significativa como factor de protección para la disminución del tiempo del segundo período del parto o para el valor del APGAR al primer minuto. Esto nos permite concluir que el uso rutinario de la episiotomía con aplicación de criterios o sin ellos no ha demostrado ser una estrategia de valor para la disminución del riesgo de desgarros grado II o mayores, ni influir en la duración del segundo período del parto por consecuencia lógica tampoco influye en el valor del APGAR al primer minuto. Por lo tanto de acuerdo al presente estudio no hay evidencia de su efecto protector.

Palabras claves: parto vaginal, riesgos, primigestas, Zacamil, episiotomía.

\section{INTRODUCCIÓN}

En el Hospital Nacional Zacamil, en el año 2006, se inició con la aplicación de criterios para la práctica de la episiotomía la cual antes de esa fecha se realizaba de forma rutinaria a discrecionalidad del obstetra tratante.

Esto reflejaba un $87 \%$ de pacientes que se les practicaba la episiotomía y posterior a la aplicación de criterios para la práctica de la Episiotomía se redujo su utilización al 39\%. Esto después del año 2006, debido a este descenso, han existido dudas e inquietudes sobre el impacto que esta medida tiene sobre las primíparas así como para la salud neonatal por lo que surge la pregunta ¿han existido cambios en el riesgo materno neonatal con una reducción en las episiotomías en casi un $50 \%$ ? y a esta interrogante genera otra ¿Realmente es necesaria? ¿Los criterios para la aplicación son necesarios?

Al revisar los criterios que se aplican según la norma establecida por el Ministerio de Salud Pública del Salvador desde el 2006 se encuentra que no hay claridad en dichos criterios y que al final siempre queda a juicio del obstetra tratante. Sin embargo estos criterios son un intento de llevar a la reflexión al profesional antes de practicar la episiotomía más que una guía clínica para la práctica de dicha intervención. Denotando la falta de consenso institucional con respecto a la práctica de la episiotomía.

El Hospital Nacional Zacamil cuenta con 26 camas en el área de ginecología y obstetricia siendo una maternidad relativamente pequeña y con moderada demanda. Además, que los médicos que 
atienden son ginecólogos graduados debido a que no hay programa de residentado para formación de ginecólogos. Lo anterior supondría una mejor calidad de atención y una mejor toma de decisión y aplicación de criterios durante la práctica de la episiotomía. Por tal razón es posible comparar dos periodos uno antes de la aplicación de criterios y el otro después de la aplicación de criterios para la práctica de la episiotomía.

La episiotomía es un procedimiento que genera controversias aun en la actualidad por lo que requiere revisión de las bases científicas que las sustentan, debido a que no hay consenso sobre el tema entre los profesionales ginecólogos. Es necesaria una investigación orientada a esclarecer esta controversia que ayude a plantear una nueva propuesta para la práctica de la episiotomía en el Hospital Nacional Zacamil.

Para lograr información confiable se realizó este estudio con el que desarrolla evidencia que sustenta de forma suficiente un cambio en la frecuencia de la práctica de la episiotomía especialmente en pacientes primíparas que son las que más sufren por esta práctica, debido a que los obstetras del hospital al preguntarles, creen que mediante la práctica de la episiotomía se disminuye el riesgo a desgarros del periné así como que favorece el bienestar del recién nacido. Pero desconocen el porqué de esta creencia ya que ambas situaciones no tiene base científica más bien son creencias que a través del tiempo se han elevado a condición de ciencia y cuando esto ocurre se crea un problema epistemológico en el que la empírea como generador de conocimiento se mal interpreta y lo que se obtiene son mitos cuentos y leyendas que causan problemas de gran magnitud para la salud pública.

$Y$ esto es un problema frecuente cuando se quiere encontrar la piedra filosofal para resolver un problema sin tener que tomar decisiones en el plano singular de cada paciente ya que esto conllevaría más trabajo intelectual y riesgos al tomar una decisión.

Por tal motivo se construyen herramientas como la episiotomía y se generalizan, de tal forma que ya no se debe de pensar en un plano singular que es el paciente, sino que estamos respaldos

por una creencia elevada a ciencia para aplicarla sin mayores análisis deductivos.

Por tal razón el planteamiento del problema de esta investigación fue: ¿cuál es la diferencia en el riesgo de complicaciones entre las primigestas que se les practica episiotomia y aquellas que no?

\section{MATERIALES Y MÉTODOS}

Tipo de estudio: de casos y controles.

Universo: todas las primigestas que verificaron parto vaginal en el año 2003 al 2004 y en el año 2007 al 2008 en el Hospital Nacional Zacamil, para el año 2003 el universo fue de 795 y para el 2007 fue de 929.

Muestra: se calculó a partir de fórmula casos y controles no pareados para una menor diferencia del $O R=1.8$ (dos colas) calculado con el programa EpiData.

Muestreo aleatorio simple se realizó posterior a la aplicación de los criterios de inclusión y exclusión a ambos universos obteniendo los siguientes resultados: Para el universo de primigestas correspondiente al año 2003-2004 que fue de 795 cumplieron con los criterios 415 en los que además se encontraban los datos necesarios en los expedientes para el estudio. La composición de estos 415 en cuanto a casos y controles fue la siguiente. Casos 190 y controles 215.

Con respecto al universo de estudio del 2007-2008 los elegibles fueron 410 de los cuales 185 cumplieron para ser casos y 225 controles, posteriormente mediante una tabla aleatoria en el programa excell se escogieron los 174 casos y controles para ambos periodos.

La significancia se evaluó en tablas 2 por 2 para comparación de dos proporciones con la prueba estadística Chi Cuadrado...Valor de p 0.05 y un nivel de confianza 95\%, con un grado de libertad para tabla 2 por 2. Valor de tabla para chi cuadrado es 3.84 


\section{Criterios de Inclusión:}

1) 19 y 35 años de edad

2) verificar parto vaginal en el Hospital Zacamil en

el año 2003 y 2007.

3) con fórmula obstétrica P000

\section{Criterios de exclusión:}

1) padecer enfermedades pélvicas articulares deformantes.

2) padecer diabetes mellitus, hipertensión arterial.

3) padecer enfermedades crónicas degenerativas como lupos eritematoso.

4) enfermedad febril aguda concurrente con el parto.

5) hemorragia del tercer trimestre, embarazos de alto riesgo.

6) sufrimiento fetal agudo.

Método de recolección y procesamiento de los datos: revisión de expedientes clínicos específicamente notas del ginecólogo, pediatra y enfermería. De cada uno de las pacientes sean casos o controles. La información de los instrumentos se vació en Epiinfo para su análisis en tablas de entrada.

\section{RESULTADOS}

Se estudiaron pacientes primigestas de dos períodos diferentes, el primer período comprende las pacientes primigestas que verificaron parto vaginal del 2003 al 2004 y el segundo período fue del 2007 al 2008 esto debido a que en el período 2003-2004 se realizaba la episiotomía de rutina y en el período 2007-2008 se realizaba la episiotomía con criterios normados.

El estudio no demostró que exista una diferencia significativa en los resultados con la realización de la episiotomía con criterios o con la realización de la episiotomía de rutina, de acuerdo a los datos comparativos siguientes:

Diferencia en el riesgo de desgarros grados en ambos períodos y valor de chicuadrado 2003-2004; 2007-2008 OR $=0.83$ chicuadrado $=0.75 \mathrm{OR}=1.1$ Chicuadrado $=0.7$
A la luz de los resultados de este estudio, desde el punto de vista estadístico aplicamos la prueba de significación de chicuadrado. Esta prueba es ampliamente utilizada en epidemiología especialmente en el análisis de tablas $2 \times 2$. Para los fines de nuestro estudio utilizamos la fórmula alternativa simplificada para chicuadrado (3). El valor de chicuadrado para tablas $2 \times 2$ con un grado de libertad, $p=0.05$ y $95 \%$ de nivel de confianza es de 3.84.(8),(9) tanto para el período 2003- 2004 y 2007-2008 el valor de chicuadrado fue de 0.75 y 0.7 respectivamente, entonces la regla de decisión es no se rechaza la hipótesis nula si el valor encontrado de chicuadrado es igual o menor a 3.84(9). Por tal motivo no hemos podido rechazar: HO. El riesgo de aumento en la magnitud de los de los daños del periné no es mayor en las primigestas sin episiotomía

Resultado que se ve reforzado al analizar la asociación a través de los valores de OR OR 0.83 para 2003-2004 y OR 1.1 para 2007-2008 En este estudio se calculó un tamaño maestral para detectar diferencias en el OR de 1,8 como mínimo, al analizar lo valores de OR para ambos períodos se observa que andan cerca de 1 , y los valores de OR de 1 se interpretan que no hay diferencia en la prevalencia de los expuestos con respecto a los no expuestos (10).

Por lo que podemos concluir que no se tiene evidencia epidemiológica que sustente una asociación protectora para el desgarro GII en primigestas con episiotomía, en el mejor de los casos es igual en ambos grupos por lo que no hay sentido en seguir practicando la episiotomía a las primigestas.

Diferencia en la duración del segundo período del parto en ambos períodos 2003-2004; 2007-2008 $\mathrm{OR}=1.12$ Chicuadrado $=0.29 \mathrm{OR}=1.13$ Chicuadrado $=\mathbf{0 . 3 0}$

El mismo análisis aplica para los resultados estadísticos de la duración de la segunda fase del parto por lo que no se puede rechazar la hipótesis nula: Ho. El riesgo de prolongación del segundo 
período del parto no es mayor en las primigestas sin episiotomía Diferencia en el valor del APGAR en el primer minuto en ambos períodos 2003-2004; 2007- 2008 OR $=1.39$ Chicuadrado $=1.65 \mathrm{OR}=1.11$ Chicuadrado $=0.16$

Con respecto a la diferencia del valor del APGAR de acuerdo al análisis previamente realizado no se tienen elementos para rechazar la hipótesis nula: Ho. El riesgo en la disminución del puntaje del APGAR no es mayor en las primigestas sin episiotomía.

\section{DISCUSIÓN}

El presente trabajo provee elementos epidemiológicos observacionales de tipo analítico que permiten valorar la utilidad de la episiotomía tanto en su modalidad rutinaria como bajo criterios normativos en la disminución del riesgo de los tres pilares en que tradicionalmente se ha fundamentado su práctica. Los cuales son:

1) Evitar desgarros del periné mayores al grado II

2) Disminución del segundo período del parto

3) Mejorar el valor del APGAR

Los elementos obtenidos en el presente trabajo no demuestran que el uso de la episiotomía ayude de forma significativa a disminuir los riesgos anteriormente mencionados independientemente que se utilice de forma rutinaria o de forma selectiva mediante la aplicación de criterios normativos.

Más allá de los números y análisis epidemiológicos si se analiza desde el punto de vista anatómico y fisiológico, la episiotomía solo es una agresión a los tejidos blandos la cual no se justifica ya que no son los tejidos blandos los que detienen el proceso fisiológico del parto vaginal, si no las estructuras óseas, las cuales no se modifican con la aplicación de la episiotomía.

Y si se toma en cuenta el grado de elasticidad de los tejidos blandos perineales se identifica que estos no se desgarran más allá del grado II, aun sin el uso de la episiotomía.

En cuanto al valor del APGAR al primer minuto como consecuencia lógica habiendo planteado que no son los tejidos blandos los que detienen o retrasan el segundo período del parto no existe fundamento basado en la evidencia que justifique el uso de la episiotomía que más que hacer un bien debido a su calidad invasiva es una agresión conscientemente realizada por los médicos.

\section{CONCLUSIONES}

A partir de los hallazgos entre casos y controles de ambos períodos estudiados, se concluye que: El uso de la episiotomía aplicando criterios normativos no difiere de los resultados obtenidos con la episiotomía de rutina.

En relación a cada objetivo del presente estudio se encuentra que:

* El uso de la episiotomía de rutina no previene los desgarros grado II del periné.

* El uso de la episiotomía no modifica significativamente la duración de la segunda fase del parto

* El uso de la episiotomía no modifica significativamente el valor del APGAR al primer minuto.

\section{BIBLIOGRAFÍA}

Ciro Martínez Bencardino, Estadística y Muestreo, esfera editores Colombia septiembre 2003

Diederick E. Grobbee, Arno W. Hoes clinical epidemiology principles, methods, and applications for clinical research. Jones \& Bartlett Publishers; 1 edition (March 20, 2008)

Dr. Amaya Galindo Alex, Maternidad del Hospital Nacional Zacamil, análisis del SIP De 5 años. Julio/2001-diciembre/2006.

Dr. Gerardo Quiroz Garza Ginecología - Obstetricia y Urología GinecológicaEpisiotomía, Mitos y Realidades, 11 27th, 2009, centro de educación prenatal y postnatal México, DF 
George M. Hall, how to write a paper fourth edition 2008, BMJ Books; 4 edition (February 19, 2008)

Guías clínicas de atención de las principales morbilidades obstétricas en el tercer nivel de atención, 2004, república de El Salvador, Ministerio de Salud Pública y asistencia social, dirección de regulación, gerencia de atención integral en la salud a la mujer.

Harold A. Kahn, Christopher T. Sempos, Statistical Methods in Epidemiology (Monographs in Epidemiology and Biostatistics), Oxford University Press, USA (August 3, 1989)

James J. Schlesselman case-control studies desing, conduct, analysis. Oxford University Press, USA; 1 edition (January 21, 1982)

John Yarnell, epidemiology and prevention a systembased approach, oxford 2007

Josep M Argimon Pallas, Josep Jiménez Villa. Métodos de investigación clínica epidemiológica tercera edición mayo 20004

León Gordis MD MPH DrPH, Epidemiology: with STUDENT CONSULT Online Access (Gordis, Epidemiology), Saunders; 4 edition (May 14, 2008).

$M$ del Pino, $M$ Puig, E Bataller, $M$ Espuña, PROTOCOLO: LESIONES PERINEALES DE ORIGEN OBSTÉTRICO: Diagnóstico, tratamiento y seguimiento. InstitutClínic de Ginecología, Obstetricia y Neonatología, Hospital Clínica de Barcelona, Última actualización: 6/4/2010

Polit, Hungler. Investigación científica en ciencias de la salud, sexta edición, McGraw hill, julio 2000

Rafael Calvo C. E.S.E Clínica de maternidad, Episiotomía-Episiorrafia, Cartagena Colombia. Octubre 2010
Ross C. Brownson, Diana B. Petitti, Applied Epidemiology: Theory to Practice, Oxford University Press, USA; 2 edition (June 29, 2006)

Ruiz, Morillo. Epidemiología clínica investigación clínica aplicada, 2004

Samuel Clavchik, Delia M, Muñoz y Marcelo Bortman.

Indicaciones de episiotomía en maternidades públicas de neuquen, argentina, Revista Panamericana de Salud Pública, vol. 4 No.1 Junio/1998

Sharon E. Straus, W. Scott Richardson, Evidence Based Medicine (3rd Edition) Churchill Livingstone; 3 edition (April 29, 2005)

Steve Selvin, Statistical Analysis of Epidemiologic Data (Monographs in Epidemiology and Biostatistics, V. 35.

Oxford University Press, USA; 3 edition (May 13, 2004) Tu Bebé 186 Algunos tratados de obstetricia Por qué se tiene que evitar la episiotomía DRA. PILARDE LA CUEVA Ginecóloga del Servicio Aragonés de la Salud. Evaluadora de la Iniciativa Hospital Amigode los Niños (IHAN) 